\title{
Centralized Testing Filenote
}

National Cancer Institute

\section{Source}

National Cancer Institute. Centralized Testing Filenote. NCI Thesaurus. Code C115504.

A notation regarding the decisions, and/or clarification of any information pertaining to a centralized test. 